\title{
DYNAMO ACTION IN MAGNETOHYDRODYNAMICS AND HALL-MAGNETOHYDRODYNAMICS
}

\author{
Pablo D. Mininni and Daniel O. Gómez ${ }^{1}$ \\ Departamento de Física, Facultad de Ciencias Exactas y Naturales, Universidad de Buenos Aires, Ciudad Universitaria, \\ 1428 Buenos Aires, Argentina; mininni@df.uba.ar \\ AND \\ Swadesh M. Mahajan \\ Institute for Fusion Studies, University of Texas, Austin, TX 78712 \\ Received 2002 October 15; accepted 2002 December 17
}

\begin{abstract}
The first direct numerical simulations of turbulent Hall dynamos are presented. The evolution of an initially weak and small-scale magnetic field in a system maintained in a stationary regime of hydrodynamic turbulence (by a stirring force at a macroscopic scale) is studied to explore the conditions for exponential growth of the magnetic energy. The Hall current is shown to have a profound effect on turbulent dynamo action; it can strongly enhance or suppress the generation of the large-scale magnetic energy depending on the relative values of the length scales of the system.
\end{abstract}

Subject headings: galaxies: magnetic fields — ISM: magnetic fields — magnetic fields — MHD stars: magnetic fields

\section{INTRODUCTION}

Dynamo activity in astrophysical environments has been historically described within the powerful but restricted framework of one-fluid magnetohydrodynamics (MHD). When the flow fields are sufficiently intense as to strongly affect or even dominate the plasma dynamics, the canonical MHD framework cannot be expected to deliver, because it does not quite distinguish the relative motions between different species.

This major shortcoming is readily rectified by including the Hall term in the generalized Ohm's law:

$$
\boldsymbol{E}=-\frac{\boldsymbol{u} \times \boldsymbol{B}}{c}+\frac{\boldsymbol{j} \times \boldsymbol{B}}{c n e}+\frac{4 \pi \eta}{c^{2}} \boldsymbol{j},
$$

where $n$ is the electron density, $e$ is the electron charge, $c$ is the speed of light, and $\eta$ is the electric resistivity. The Hall effect, represented by the $\boldsymbol{j} \times \boldsymbol{B}$ term, is one of the more important manifestations of the velocity difference between species. For a two-species quasi-neutral electron-ion plasma, the current $\boldsymbol{j}$ is indeed a direct measure of the relative electron-ion velocity. Inclusion of the Hall term leads to the so-called Hall-MHD equations, which display, among other properties, the freezing of the magnetic field to the electron flow (in the nondissipative limit) rather than to the bulk velocity field.

The astrophysical systems in which the Hall effect might be important are relatively well known. It has a profound influence, for instance, on the magnetic field dynamics in dense molecular clouds (Wardle \& Ng 1999), and in accretion disks (especially in protostellar disks) it strongly affects the Balbus-Hawley magnetorotational instability (Wardle 1999; Balbus \& Terquem 2001). Norman \& Heyvaerts (1985) also noted that the Hall effect may become significant during star formation, and its relevance to compact objects such as white dwarfs and neutron stars (Urpin \& Yakovlev

\footnotetext{
${ }^{1}$ Also at Instituto de Astronomía y Física del Espacio, Ciudad Universitaria, 1428 Buenos Aires, Argentina.
}

1980; Shalybkov \& Urpin 1997; Potekhin 1999) has also been discussed. In addition, it can play a crucial role in the generation and evolution of magnetic fields in the early universe (Tajima et al. 1992).

To assess the relevance of the Hall term in these scenarios, we follow Balbus \& Terquem (2001) and denote the terms on the right-hand side of Ohm's law (eq. [1]) from left to right as $I$ (inductive), $O$ (ohmic), and $H$ (Hall). We assume charge neutrality and compare these terms by defining the ratios

$$
\begin{gathered}
\frac{H}{O}=\frac{\omega_{e}}{\nu_{e}}, \\
\frac{H}{I}=\frac{\omega_{e}}{\nu_{e} \operatorname{Re}_{m}} .
\end{gathered}
$$

Here, $\operatorname{Re}_{m}=U_{\mathrm{A}} L / \eta$ is the magnetic Reynolds number, $U_{\mathrm{A}}=B /(4 \pi \rho)^{1 / 2}$ is a characteristic Alfvénic speed, $\rho$ is the mass density, and $L$ is a characteristic length. The electron cyclotron frequency $\omega_{e}$ and the collision frequency $\nu_{e}$ are given by

$$
\begin{gathered}
\omega_{e}=\frac{e B}{m c}, \\
\nu_{e}=\frac{4 \pi e^{2} n \eta}{m c^{2}},
\end{gathered}
$$

where $m$ is the electron mass. In a protostellar disk, considering a density of neutrals $n_{N} \approx 10^{13} \mathrm{~cm}^{-3}$, a mass density $\rho=2.33 n_{N} m_{p}$ ( $m_{p}$ : proton mass), equipartition between magnetic and thermal energy $U_{\mathrm{A}} \approx 0.429 \mathrm{kTm}_{p}^{-1}$ ( $T$ : temperature; $k$ : Boltzmann constant), and $\eta=234 n_{N} n^{-1} T^{1 / 2} \mathrm{~cm}^{2}$ $\mathrm{s}^{-1}$ (Balbus \& Terquem 2001) yields $H / O \approx 10^{2}$. Therefore, the Hall effect is more important than ohmic losses and must be relevant in this scenario. Considering a characteristic length of $1 \mathrm{AU}$ and $n \approx 10^{5} \mathrm{~cm}^{-3}$ (Shu et al. 1994), we obtain $H / I \approx 10^{-4}$. In a dwarf nova disk, if $T \approx 1500 \mathrm{~K}$, $n_{N} \approx 10^{16} \mathrm{~cm}^{-3}$, and $L \approx 10^{10} \mathrm{~cm}$, then $H / I \approx 1$ (Sano \& Stone 2002) and can be even larger in some regions of protoplanetary disks. Finally, in the crust of a neutron star with 
$B \approx 10^{12} \mathrm{G}$, we find $H / O \approx 10^{3}$ (Hollerbach \& Rüdiger 2002). We come back to the relevance of these numbers in $\S 6$.

In spite of this widespread appreciation that the Hall effect may be highly relevant, it has not been included in most studies devoted to the production of the macroscopic magnetic fields observed in such objects. A few exceptions are studies by Heintzmann (1983) and Galanti, Kleeorin, \& Rogachevskii (1994), focused on particular geometries and showing either suppression or enhancement of dynamo action depending on external parameters, an analysis of the effect of turbulent dynamo action on magnetic helicity generation (Ji 1999), and a recent general closure proposed to compute the contribution of the Hall term to the $\alpha$-effect (Mininni, Gómez, \& Mahajan 2002). Preliminary results from the latter study show that both large suppression or enhancement of dynamo action (as compared to standard MHD) are possible, depending on parameters that describe the state of the system. It was also found that saturation in Hall dynamos might be reached by a mechanism different from the standard Alfvénic suppression.

In the present work, we present preliminary results of a numerical simulation of Hall MHD to study dynamos with strong kinetic helical forcing. To the best of our knowledge, these are the first numerical simulations showing turbulent dynamo action in this broader framework.

When the Hall term is switched off, we reproduce the results obtained by other authors (Meneguzzi, Frisch, \& Pouquet 1981; Cattaneo \& Hughes 1996; Brandenburg 2001 and references therein). We use the term "Hall-MHD dynamo " to distinguish the new dynamo from the standard classical MHD dynamo. In our simulation, following the previous authors (Meneguzzi et al. 1981; Brandenburg 2001), we generate and maintain a stationary background of hydrodynamic turbulence by applying an external force operating at a macroscopic scale $L_{\text {force }}$. In this setting we introduce a very weak "seed" magnetic field at a microscopic scale $L_{\text {seed }}$ and study its temporal evolution, paying particular attention to solutions with exponential growth of the magnetic energy and other relevant aspects associated with dynamo activity. From an inspection of Ohm's law (see eq. [3]), it follows that the Hall term becomes nonnegligible with respect to the induction term at length scales of the order of the collisionless ion skin depth, which we can denote in a fully ionized plasma as $L_{\mathrm{Hall}}=c / \omega_{\mathrm{pi}}(c$ : speed of light; $\omega_{\mathrm{pi}}$ : ion plasma frequency). For the problem at hand, three distinct dynamo regimes emerge: (1) When the Hall length scale $L_{\text {Hall }}$ falls within the forcing and seed length scales (i.e., $L_{\text {seed }} \leq L_{\text {Hall }} \leq L_{\text {force }}$ ), the system finds itself in the "Hall-enhanced" regime with a dramatic enhancement of dynamo activity. (2) The range $L_{\text {Hall }}>L_{\text {force }}$, on the other hand, defines the "Hall-suppressed" regime, with an important reduction in dynamo action (compared to the MHD case). (3) For $L_{\mathrm{Hall}}<L_{\text {seed }}$, as $L_{\mathrm{Hall}}$ approaches the dissipation scale, the Hall effect becomes gradually negligible, and we asymptotically recover the standard MHD dynamo.

In $\S 2$ we briefly introduce the Hall-MHD equations and describe their relevant properties. A description of the numerical code developed to integrate these equations is given in $\S 3$. In $\S 4$ we benchmark the code by comparing our results (with the Hall term switched off) with the results from previous studies. The effects of the Hall term at different values of the Hall length $L_{\mathrm{Hall}}$ are reported and high- lighted in $\S 5$. Finally, in $\S 6$ we summarize the conclusions of the present preliminary study.

\section{THE HALL-MHD SYSTEM}

Incompressible Hall-MHD is described by modified induction and the Navier-Stokes equation:

$$
\begin{gathered}
\frac{\partial \boldsymbol{B}}{\partial t}=\nabla \times[(\boldsymbol{U}-\epsilon \boldsymbol{\nabla} \times \boldsymbol{B}) \times \boldsymbol{B}]+\eta \nabla^{2} \boldsymbol{B}, \\
\frac{\partial \boldsymbol{U}}{\partial t}=-(\boldsymbol{U} \cdot \boldsymbol{\nabla}) \boldsymbol{U}+(\boldsymbol{B} \cdot \boldsymbol{\nabla}) \boldsymbol{B}-\boldsymbol{\nabla}\left(P+\frac{B^{2}}{2}\right)+\nu \nabla^{2} \boldsymbol{U},
\end{gathered}
$$

with the additional constraints $\boldsymbol{\nabla} \cdot \boldsymbol{U}=\boldsymbol{\nabla} \cdot \boldsymbol{B}=0$. The velocity and magnetic fields are expressed in units of a characteristic speed $U_{0}, \epsilon$ measures the relative strength of the Hall effect, $\eta$ is the magnetic diffusivity, and $\nu$ is the kinematic viscosity. Note that, considering the characteristic speed $U_{0}$ and the characteristic Alfvénic speed $U_{\mathrm{A}}, \epsilon$ can be written as

$$
\epsilon=\frac{L_{\mathrm{Hall}}}{L_{0}},
$$

where $L_{0}$ is a characteristic length scale (the size of the box in our simulations: $L_{0}=1$ ), and

$$
L_{\text {Hall }}=\frac{c}{\omega_{\mathrm{pi}}} \frac{U_{\mathrm{A}}}{U_{0}}
$$

is the Hall length scale. In particular, we are free to choose $U_{0}=U_{\mathrm{A}}$ as our characteristic velocity reducing $L_{\text {Hall }}$ to the ion skin depth. Equation (9) is valid in a fully ionized plasma. We work under this assumption without loss of generality; in the general case, the values of $L_{\mathrm{Hall}}$ and $\epsilon$ must be obtained from equations (2) and (3).

This system has three well-known ideal $(\eta=\nu=0)$ quadratic invariants:

$$
\begin{gathered}
E=\frac{1}{2} \int\left(U^{2}+B^{2}\right) d V, \\
H_{m}=\frac{1}{2} \int \boldsymbol{A} \cdot \boldsymbol{B} d V, \\
K=\frac{1}{2} \int(\boldsymbol{B}+\epsilon \boldsymbol{\omega}) \cdot(\boldsymbol{A}+\epsilon \boldsymbol{U}) d V,
\end{gathered}
$$

where $E$ is the energy, $H_{m}$ is the magnetic helicity, and $K$ is the hybrid helicity, which replaces the cross-helicity from magnetohydrodynamics. Here $\boldsymbol{A}$ is the vector potential, defined by $\boldsymbol{B}=\boldsymbol{\nabla} \times \boldsymbol{A}$. Conservation of these ideal invariants during the evolution of the system provides a check on the simulation.

We have already delineated three distinct Hall dynamo regimes defined by the relative values of the four basic scales of the system, the macroscopic scale $L_{\text {force }}$ for the external driver, the microscopic scale $L_{\text {seed }}$ associated with the seed magnetic field, the dissipation scale, and the Hall length $L_{\text {Hall }}$. A fourth regime could be added in which both $L_{\text {force }}$ and $L_{\text {seed }}$ are smaller than $L_{\mathrm{Hall}}$ and in which the Hall effect should be important at all relevant scales. For instance, in Mininni, Gómez, \& Mahajan (2003), we studied the change in dynamo waves for $L_{\text {force }} \gg L_{\text {seed }} \approx L_{\text {Hall }}$.

Before embarking on the simulation path, it is instructive to discuss aspects of the semianalytical work of Mininni et al. (2003). It was shown that the expression for the $\alpha$-effect 
in the presence of the Hall effect is modified according to

$$
\begin{aligned}
\alpha= & \frac{\tau}{3}\left(-\left\langle\boldsymbol{u}_{0}^{e} \cdot \nabla \times \boldsymbol{u}_{0}^{e}\right\rangle+\left\langle\boldsymbol{b}_{0} \cdot \nabla \times \boldsymbol{b}_{0}\right\rangle\right. \\
& \left.-\epsilon\left\langle\boldsymbol{b}_{0} \cdot \nabla \times \nabla \times \boldsymbol{u}_{0}^{e}\right\rangle\right),
\end{aligned}
$$

where $\boldsymbol{u}^{e} \equiv \boldsymbol{u}-\epsilon \boldsymbol{\nabla} \times \boldsymbol{b}$ is the small-scale electron flow velocity, and $\boldsymbol{u}_{0}$ and $\boldsymbol{b}_{0}$ are respectively the small-scale velocity and magnetic fields. The coefficient $\tau$ is a typical correlation time for the turbulent small-scale motions. This general expression (eq. [13]) differs from the classical result in two ways: it replaces the kinetic helicity (of the bulk motion) by the helicity of the electron flow and contains an extra term due to the Hall current in the microscale. A nontrivial consequence of the latter is that while the original $\alpha$-coefficient of Pouquet, Frisch, \& Leorat (1976) is 0 for a pure Alfvénic state $\boldsymbol{u}= \pm \boldsymbol{b}$ (Gruzinov \& Diamond 1994), the one corresponding to equation (13) is not. Note that this expression is highly nonlinear in the magnetic field and the electric current, since the electron flow velocity is strongly affected during the growth of the magnetic field. The expression is also nonlinear in the amplitude of the Hall effect $(\epsilon)$, since $\boldsymbol{u}_{0}^{e}$ is linear in $\epsilon$. Therefore, the expression in equation (13) is quadratic in $\epsilon$, and the dependence of the efficiency of the $\alpha$ effect on the amplitude of the Hall effect is not necessarily monotonic.

Since the classical $\alpha$-effect is dominated by kinetic helicity, many MHD simulations were done by imposing helical forcing in the Navier-Stokes equation (Meneguzzi et al. 1981; Brandenburg 2001). On the other hand, the HallMHD $\alpha$-effect is expected to be dominated by the electronic kinetic helicity (see eq. [13]), which can be quite different from the kinetic helicity. In the present work we use helical kinetic forcing for a comparison between classical MHD and Hall-MHD dynamo actions.

\section{THE CODE}

We integrated the Hall-MHD system (eq. [7]) in a cubic box with periodic boundary conditions. Spatial derivatives were computed using a pseudospectral scheme (Orzag \& Patterson 1972), with the two-thirds rule to control aliasing truncation errors. The equations were evolved in time using a Runge-Kutta method of order 2. The total pressure $\left(P+B^{2} / 2\right)$ was computed in a self-consistent fashion at each time step to ensure the incompressibility condition $\boldsymbol{\nabla} \cdot \boldsymbol{U}=0$ (Canuto et al. 1988). To satisfy the divergencefree condition for the magnetic field, the induction equation was replaced by an equation for the vector potential:

$$
\frac{\partial \boldsymbol{A}}{\partial t}=(\boldsymbol{U}-\epsilon \boldsymbol{\nabla} \times \boldsymbol{B}) \times \boldsymbol{B}-\nabla \phi+\eta \nabla^{2} \boldsymbol{A},
$$

where $\phi$ was computed at each time step to satisfy $\boldsymbol{\nabla} \cdot \boldsymbol{A}=0$. This particular choice of gauge (which does not imply any loss of generality in the solutions for $\boldsymbol{B}$ ) was found to improve the stability of the code in runs dominated by the Hall effect.

We present results from six different runs, with $64 \times 64 \times 64$ spatial grid points and $\epsilon=0,0.066,0.1,0.2$, 0.5 , and 1. All runs were made with magnetic Prandtl number $\operatorname{Pr}_{m}=1(\nu=\eta=0.05)$.

The Navier-Stokes equation was subjected to a stationary helical forcing $\boldsymbol{F}$ (namely, an $A B C$ flow with

$$
\begin{aligned}
A=B=C=1) & \\
\boldsymbol{F}= & F_{0}\left\{\left[\cos \left(k_{\text {force }} y\right)+\sin \left(k_{\text {force }} z\right)\right] \hat{\boldsymbol{x}}\right. \\
& +\left[\sin \left(k_{\text {force }} x\right)+\cos \left(k_{\text {force }} z\right)\right] \hat{\boldsymbol{y}} \\
& \left.+\left[\cos \left(k_{\text {force }} x\right)+\sin \left(k_{\text {force }} y\right)\right] \hat{\boldsymbol{z}}\right\},
\end{aligned}
$$

centered at $k_{\text {force }}=3$. Therefore, the helicity density of the external force satisfies

$$
\boldsymbol{F} \cdot \boldsymbol{\nabla} \times \boldsymbol{F}=k_{\text {force }}|\boldsymbol{F}|^{2} .
$$

First, a hydrodynamic simulation was conducted to reach a turbulent stationary state. Initially, the velocity was taken to be $0(\boldsymbol{u}=0)$, and a nonhelical small magnetic seed was introduced, with $E_{m} \approx 10^{-5} E_{k}$ (where $E_{k}=\left\langle U^{2}\right\rangle / 2$ and $E_{m}=\left\langle B^{2}\right\rangle / 2$ are respectively the kinetic and magnetic energy). The initial magnetic seed was generated by a $\delta$ correlated vector potential centered at $k_{\text {seed }}=13$, which yields a magnetic energy spectrum $E_{m}(k) \approx k^{4}$. The magnetic helicity of the initial seed was chosen to be 0 . The run was continued with the same helical forcing in the NavierStokes equation, to study the growth of the magnetic energy due to dynamo action. For a meaningful comparison, all the runs were carried out with the same Reynolds numbers, the same initial kinetic energy, and the same magnetic seed.

\section{RESULTS FOR MHD DYNAMOS}

In Figure 1 we show the magnetic and kinetic energy as a function of time for a pure MHD run $(\epsilon=0)$. The kinetic helicity $H_{k}=\langle\boldsymbol{U} \cdot \boldsymbol{\nabla} \times \boldsymbol{U}\rangle$, the magnetic helicity $H_{m}=\langle\boldsymbol{A} \cdot \boldsymbol{B}\rangle$, and the current helicity $H_{j}=\langle\boldsymbol{B} \cdot \boldsymbol{J}\rangle$ (where $\boldsymbol{J}=\boldsymbol{\nabla} \times \boldsymbol{B})$ are displayed in Figure 2. Note that during the dynamo process, the magnetic helicity is generated with a sign opposite to that of the kinetic helicity, which therefore has the same sign as the classical $\alpha$-coefficient. On the other hand, the helicity associated with the current grows with the same sign as the kinetic helicity.

The primary reason for the generation of magnetic helicity lies in the conservation of magnetic helicity coupled with the dissipative events needed for the dynamo to work. Since the external "forcing" is restricted to the NavierStokes equation, the net magnetic helicity is conserved, except for magnetic diffusion at small scales:

$$
\frac{d H_{m}}{d t}=-2 \eta \int \boldsymbol{J} \cdot \boldsymbol{B} d^{3} x
$$

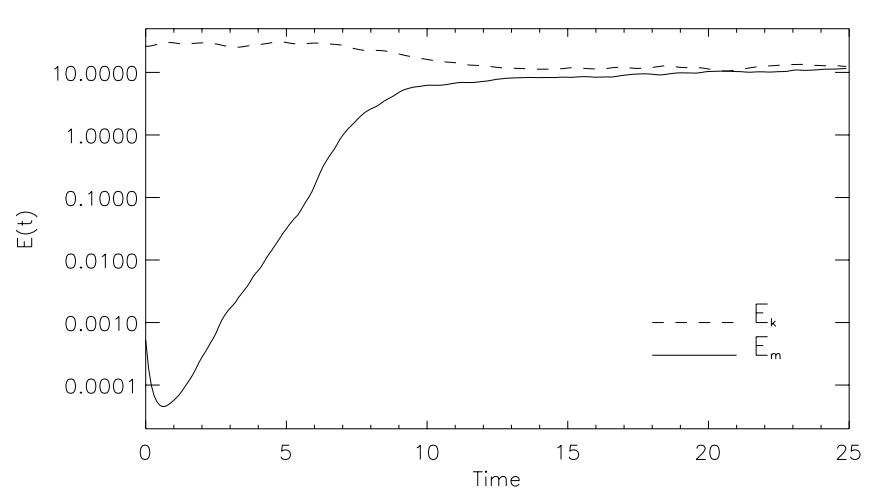

FIG. 1.-Magnetic energy $E_{m}$ and kinetic energy $E_{k}$ as a function of time $(\epsilon=0)$. 


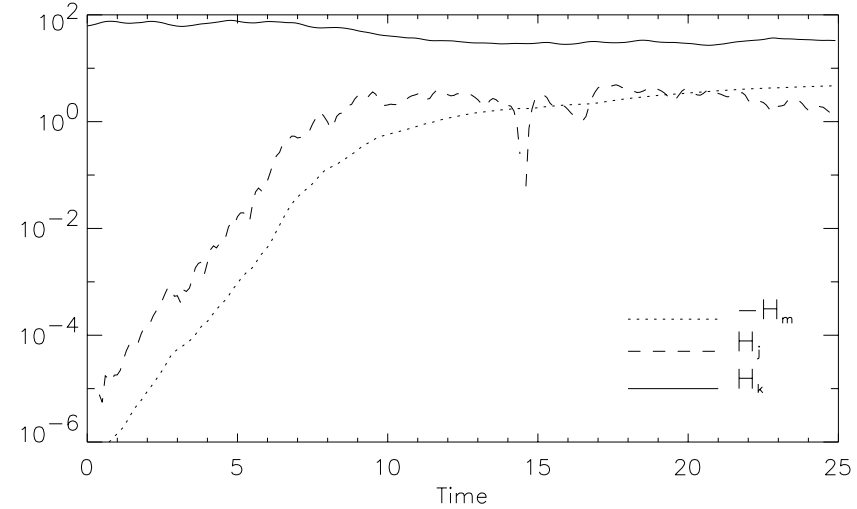

FIG. 2.-Kinetic helicity $H_{k}$, current helicity $H_{j}$, and magnetic helicity $-H_{m}(\epsilon=0)$.

However, at large scales the $\alpha$-effect can change the helicity of the mean field (Seehafer 1996). For the mean magnetic helicity we obtain

$$
\frac{d \overline{H_{m}}}{d t}=2 \int\left(\alpha \bar{B}^{2}-\eta_{\mathrm{eff}} \overline{\boldsymbol{J}} \cdot \overline{\boldsymbol{B}}\right) d^{3} x,
$$

where the overbar denotes the mean field and $\eta_{\text {eff }}$ is the magnetic diffusivity $\eta$ plus the turbulent diffusivity. Equation (18) represents a transfer of magnetic helicity from small scales to large scales. The mean field helicity has the same sign as the $\alpha$-coefficient; our results (see Fig. 2) are in good agreement with this relation. Note, however, that this relation could be affected by turbulent diffusivity.

Since the net magnetic helicity is conserved, and the original helicity is 0 , where does this mean (large scale) helicity come from? The pathway is rather straightforward: First, the $\alpha$-effect creates equal but opposite amounts of magnetic helicity in the micro- and macroscales. Then, the dissipation (diffusion) preferentially destroys the short-scale magnetic helicity in reconnection events, leaving a net helicity of opposite sign at large scales (Brandenburg 2001). This effect is expected to decrease as the magnetic Reynolds number $\mathrm{Re}_{m}$ increases.

After an initial stage with exponential growth (which can be considered a kinematic dynamo stage), the magnetic energy saturates and reaches equipartition with the kinetic energy (see Fig. 1). Figure 3 shows the kinetic and magnetic spectra at different times. Note that during the first few time steps, the original $k^{4}$ power spectrum of the initial seed (corresponding to a $\delta$-correlated vector potential in $k_{\text {seed }}=13$ ) is significantly deformed until the magnetic energy is almost equally distributed at all length scales. Then, the spectrum starts to grow at all scales at almost the same rate. This result is in good agreement with previous simulations and theoretical predictions of MHD dynamo action (Kazantsev 1968; Brandenburg 2001). Finally, the magnetic energy in each scale reaches equipartition with the kinetic energy, with some regions where the magnetic energy is even somewhat larger than the kinetic energy.

The resulting emergence of a large-scale field can be clearly seen both in the spectrum and also in slices of the cube at different times, showing the density of the magnetic field intensity (Fig. 4).

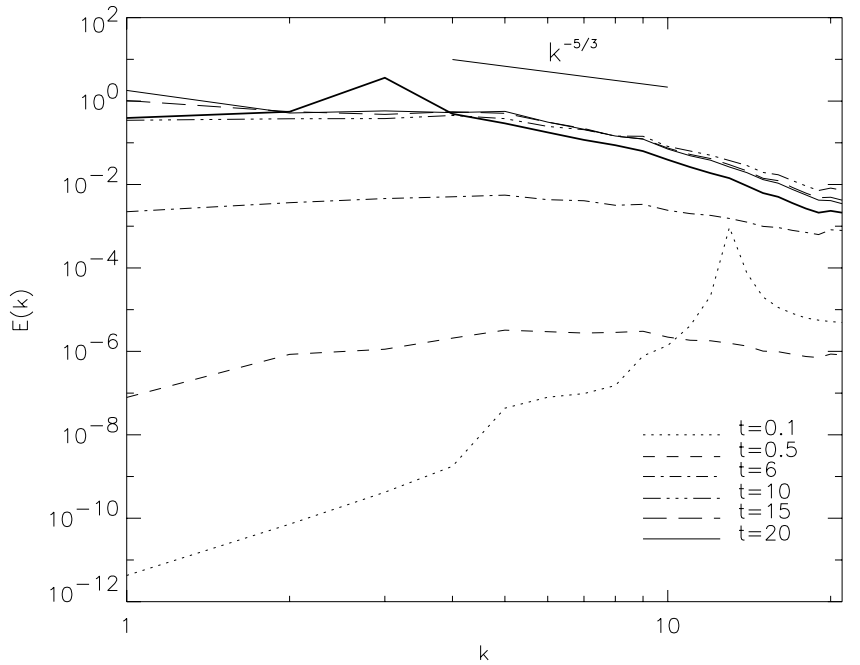

FIG. 3.-Mean kinetic energy spectrum (thick line) and magnetic energy spectrum at different times $(\epsilon=0)$. The Kolmogorov slope is shown only as a reference.

\section{RESULTS FROM HALL-MHD DYNAMOS}

To quantitatively assess the role of the Hall effect on dynamo action, we display results from six runs with different values of the parameter $\epsilon$ : the MHD run $(\epsilon=0)$ and five Hall-MHD runs with $\epsilon=0.066,0.1,0.2,0.5$, and 1 . The Hall inverse length scale for these runs is measured by $k_{\text {Hall }}=15,10,5,2$, and 1 , respectively. All length scales smaller than the Hall scale are expected to be strongly affected by the Hall effect. The magnetic seed is initially located at $k_{\text {seed }}=13$, and the Kolmogorov dissipation scale $\left[k_{\nu}=\left(\left\langle\omega^{2}\right\rangle / \nu^{2}\right)^{1 / 4}\right]$ in all runs is $k_{\nu} \approx 20$. Note that these values of $\epsilon$ show three different possible scenarios in a turbulent dynamo: (1) the MHD dynamo, (2) a Hall-dominated microscale dynamo, where the effect is relevant in only a fraction of the scales involved $(0<\epsilon<1)$, and (3) the massive Hall-MHD dynamo, where the Hall effect is relevant at all length scales $(\epsilon \geq 1)$.

Figure 5 shows the kinetic and magnetic energy as a function of time for the MHD run and two different Hall-MHD runs with $\epsilon=0.5$ and 1 . For early times, the evolution of magnetic energy in MHD and Hall-MHD systems is similar. The exponential growth of the magnetic energy in the three runs has the same rate. However, this exponential stage saturates faster in the Hall-MHD runs. As a result, equipartition is not reached in these runs. This result is in good agreement with equation (13), which predicts a change in the growth rate as the magnetic field grows.

Figure 6 shows the same results for runs with $\epsilon=0.066$ and 0.1 . Note that for these values, the system does reach equipartition between its kinetic and magnetic energies. Moreover, for $\epsilon=0.1$, the magnetic energy saturates at a value that is approximately $80 \%$ bigger than the MHD value; the saturation energy is about $100 \%$ bigger for $\epsilon=0.2$. On the other hand, for $\epsilon=0.066$, the magnetic energy is rather close to the MHD run. In this case, $k_{\text {Hall }}=15$ is smaller than $k_{\text {seed }}$ and closer to the dissipation scale $k_{\nu}$. It is not surprising, therefore, that as $\epsilon$ goes to 0 , the Hall-MHD results converge to the classical MHD dynamo.

When equipartition is finally attained, the sum of the magnetic and the kinetic energy is not always equal to the 

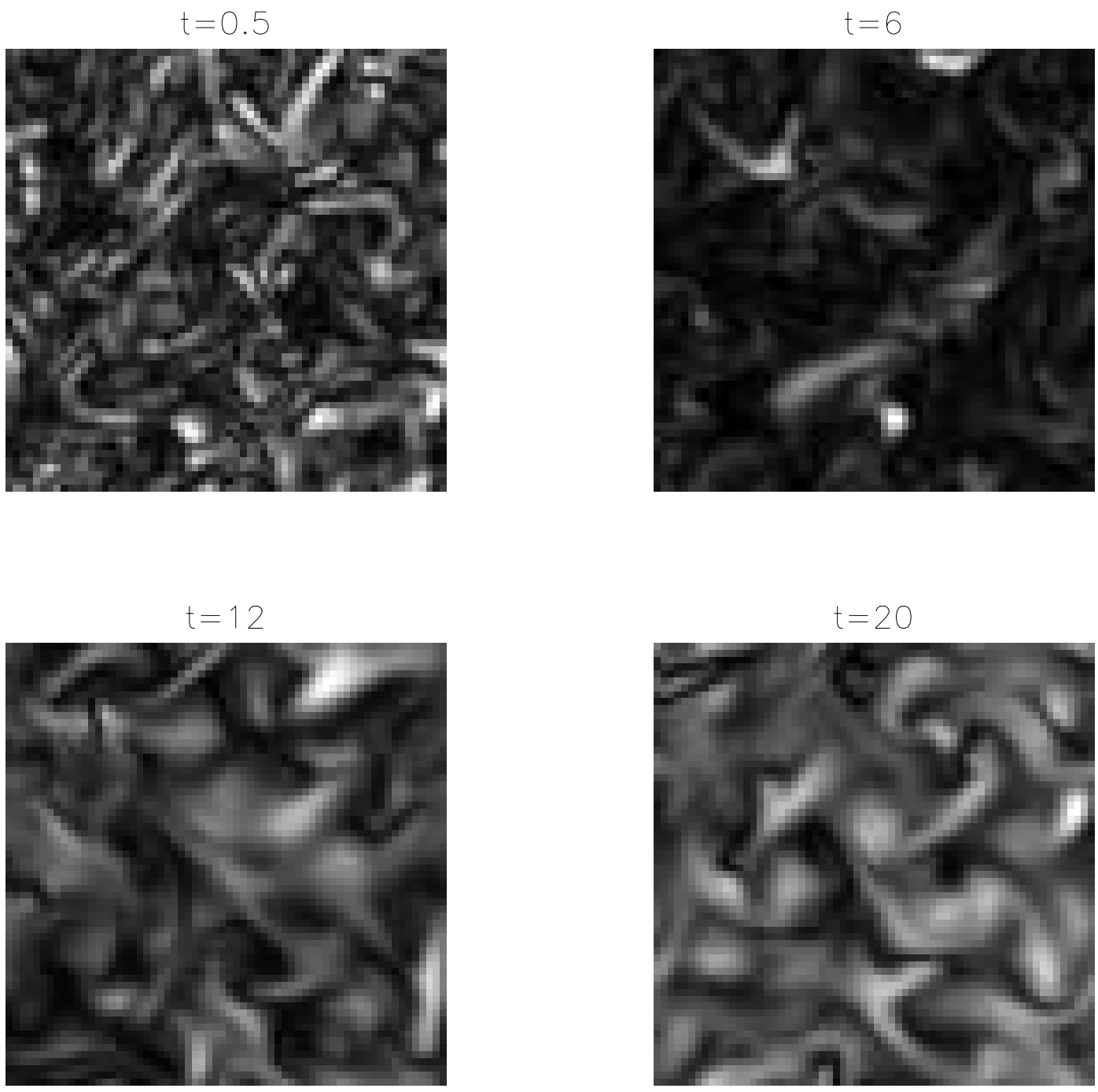

FIG. 4.- Slices of $B_{\text {rms }}=\left(E_{m}\right)^{1 / 2}$ at different times $(\epsilon=0)$

initial kinetic energy. This is related to the fact that when the magnetic seed is introduced, a new channel for energy dissipation (namely, Joule dissipation) arises:

$$
\frac{d E}{d t}=-2 \nu \Omega-\eta \int J^{2} d^{3} x
$$

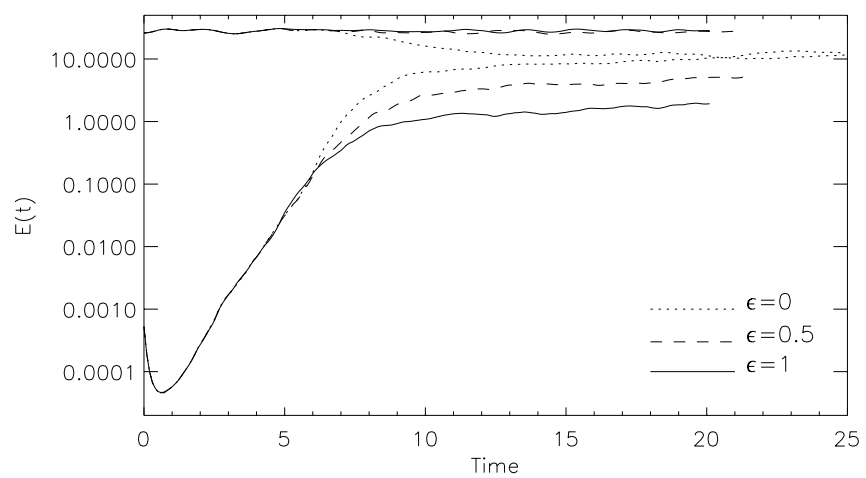

FIG. 5.-Magnetic (bottom curves) and kinetic (top curves) energy as a function of time for three different runs with $\epsilon=1,0.5$, and 0 . When $\epsilon=1$, the magnetic energy is 1 order of magnitude smaller than the MHD case. Some lines were not drawn up to the same time for clarity. where $\Omega$ is the enstrophy. It is found that the final energy reached for some of the Hall-MHD runs is larger than the value obtained for the MHD run, revealing that Hall-MHD dynamos can be more efficient (in the sense that they generate more magnetic energy) and at the same time reach equipartition. For this argument to be valid, the mean value of the current must decrease as $\epsilon$ increases, as is shown below.

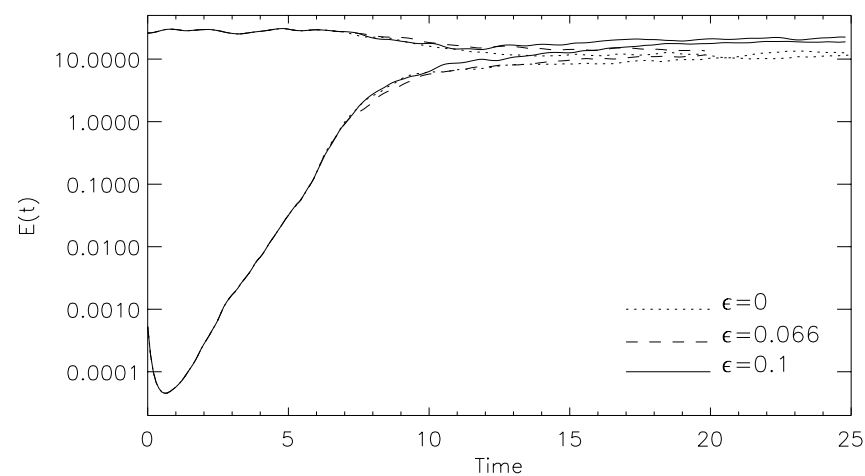

FIG. 6.-Magnetic (bottom curves) and kinetic (top curves) energy as a function of time for three different runs with $\epsilon=0,0.1$, and 0.066 . When $\epsilon=0.1$, the magnetic energy is $80 \%$ bigger than in the MHD case. 


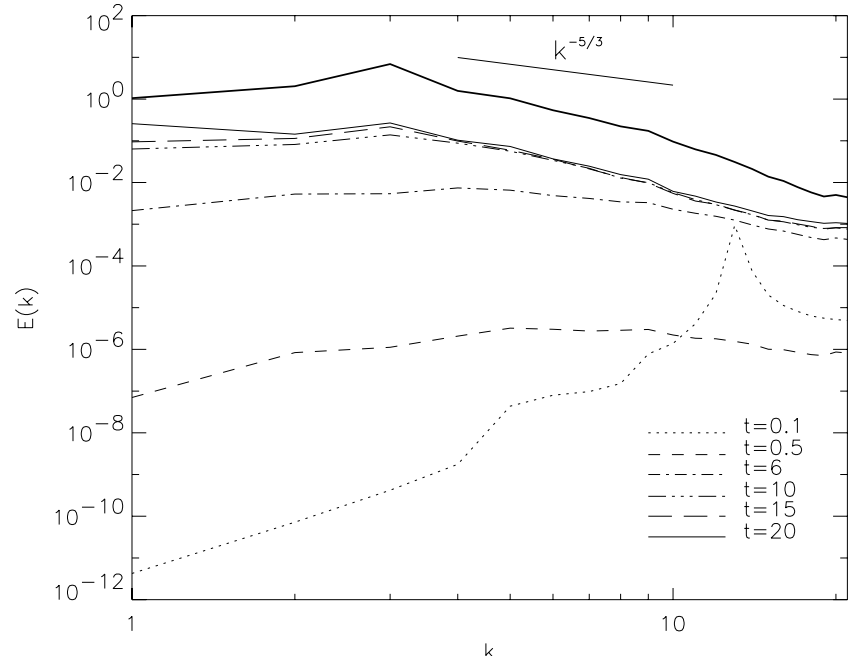

FIG. 7.-Mean kinetic energy spectrum (thick line) and magnetic energy spectrum at different times $(\epsilon=1)$.
Figure 7 shows the kinetic and magnetic energy spectra at different times for $\epsilon=1$. During the first few steps, the evolution is similar to the MHD run, with the entire magnetic spectrum growing at almost the same rate. There is a difference, however, in that the large-scale magnetic field is slightly larger than the MHD counterpart. Finally, the magnetic energy saturates at a smaller value than the one expected for equipartition. It is worth noting that the saturation is reached at all scales with almost the same $E_{m} / E_{k}$ ratio. Figure 8 shows the evolution of the magnetic field intensity in slices of the cube for this run.

Figure 9 shows the kinetic and magnetic energy spectra for different times for the run with $\epsilon=0.1$. Here the equipartition is reached at intermediate scales; there are regions (at both large and small scales) where the magnetic energy is even somewhat larger than the kinetic energy. Note that the spectrum obtained is similar to the MHD spectrum at large and small scales. However, the shape of the spectrum is slightly different at intermediate scales. The emergence of a large-scale field can also be clearly seen in Figure 10, which shows the evolution of magnetic field intensity in slices of the cube.
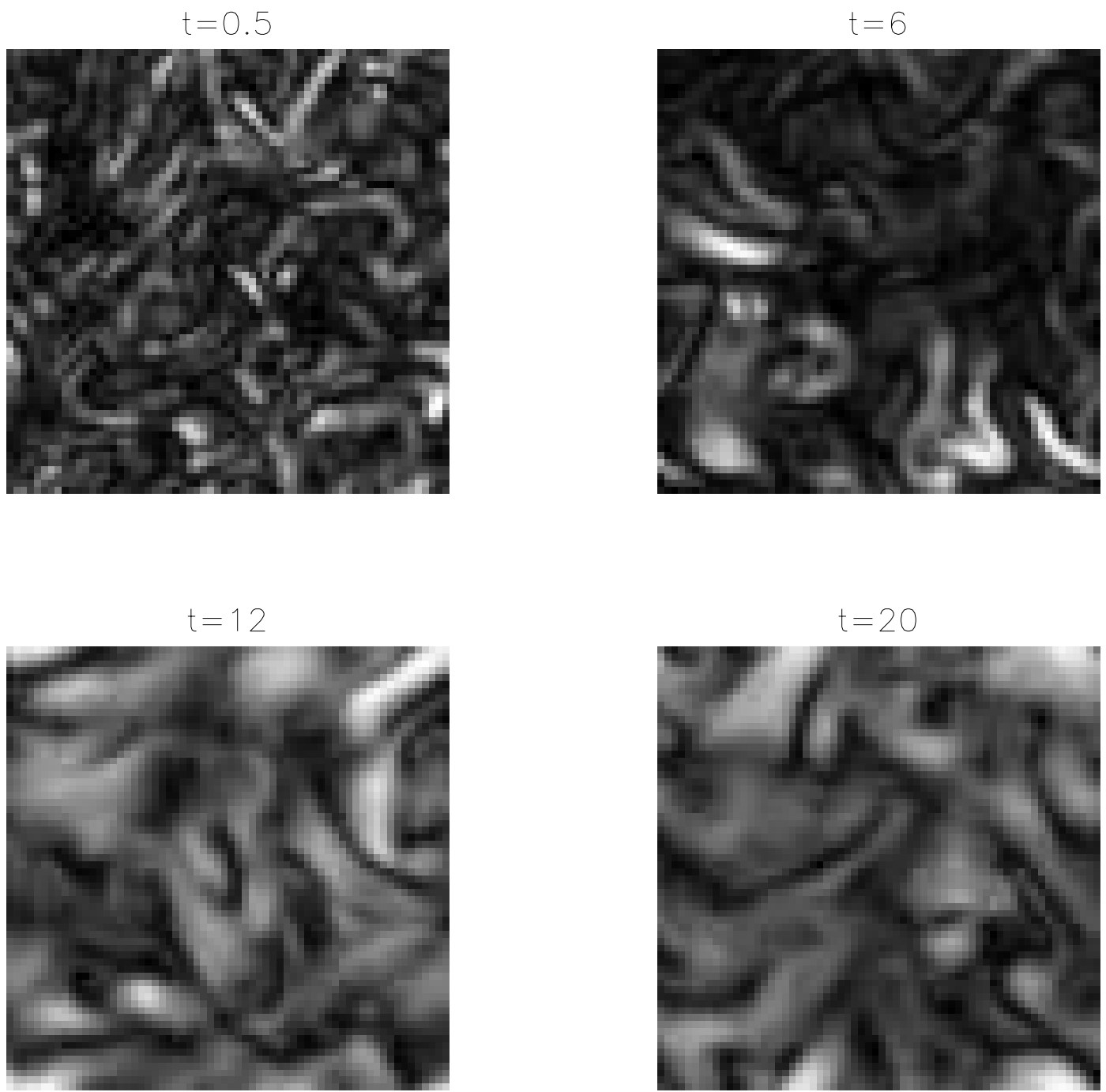

Fig. 8. - Slices of $B_{\mathrm{rms}}$ at different times $(\epsilon=1)$ 


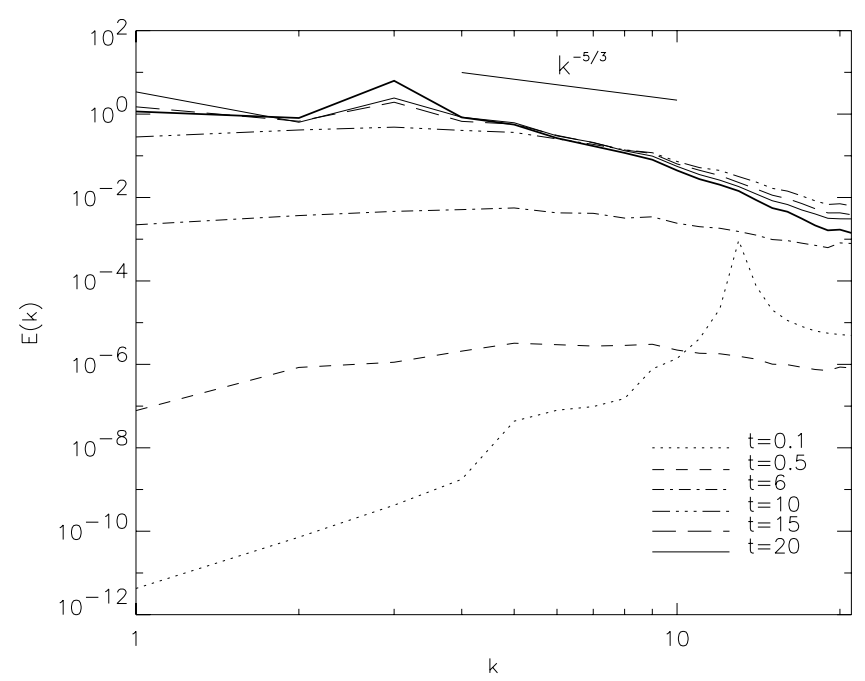

FIG. 9.-Mean kinetic energy spectrum (thick line) and magnetic energy spectrum at different times $(\epsilon=0.1)$.
In Figure 11 we plot $\left\langle J^{2}\right\rangle$ as a function of time for three different runs. Note that as the Hall term $(\epsilon)$ increases, $J$ decreases. In addition, $\boldsymbol{J}$ has a finer structure as $\epsilon$ increases. Therefore, the magnetic dissipation scale $\left[k_{\eta}=\left(\left\langle J^{2}\right\rangle / \eta^{2}\right)^{1 / 4}\right]$ is smaller as $\epsilon$ increases, and dissipation should be expected to take place at larger scales. In addition, the electronic kinetic energy decreases as the Hall term becomes larger (see Fig. 12). In all Hall-MHD runs, the electronic kinetic helicity decreases when the magnetic energy increases. In addition, note that the electronic kinetic helicity changes sign at about the same time the magnetic field saturates. This simultaneity between the sign reversal in the electronic kinetic helicity and the saturation of the magnetic field was observed in all Hall-MHD runs that did not reach equipartition (Fig. 13). In all these figures, the behavior of a typical stationary turbulent regime can be observed, corresponding to the asymptotic relaxation to constant values for these global (i.e., spatially integrated) physical quantities. The presence of spikes superposed on these values can also be observed, which is a consequence of the intermittent behavior of turbulent systems.
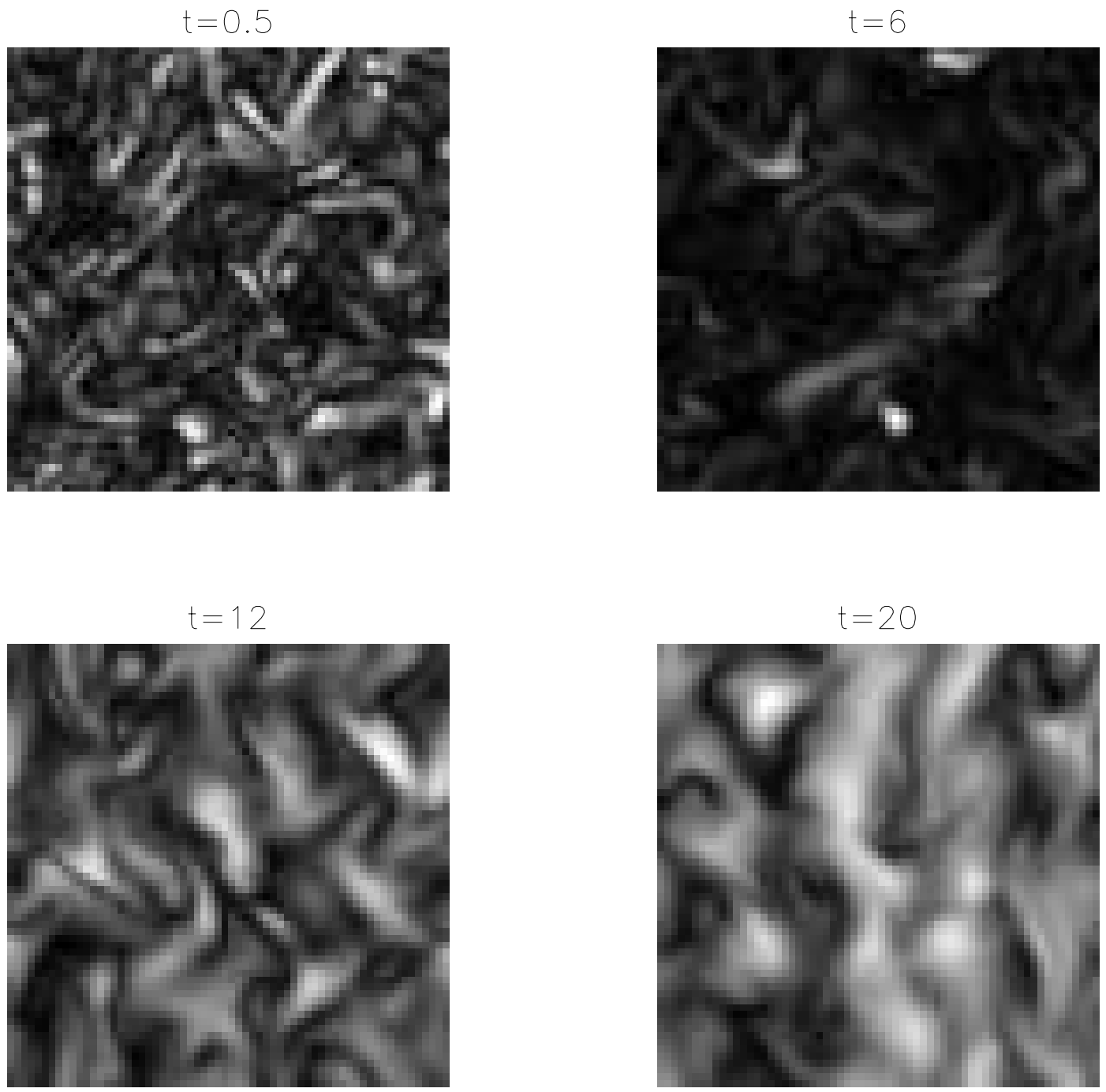

FIG. 10.- Slices of $B_{\mathrm{rms}}$ at different times $(\epsilon=0.1)$ 


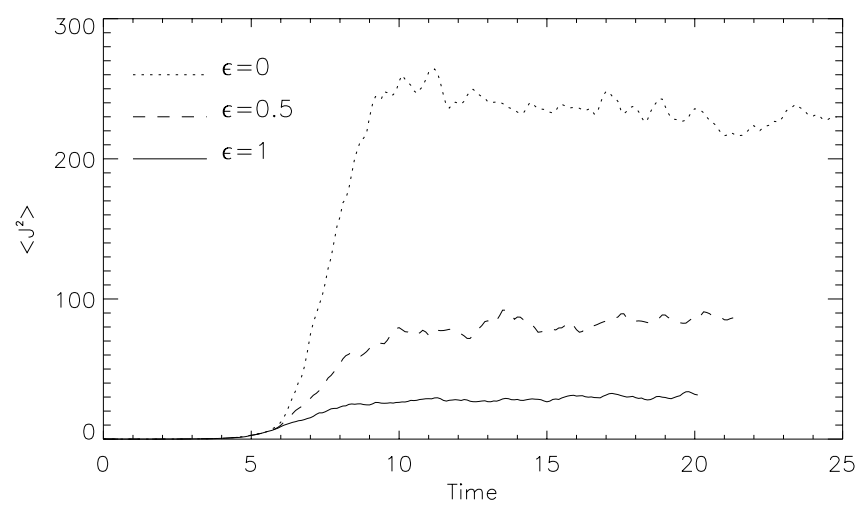

FIG. 11.-Plot of $\left\langle J^{2}\right\rangle$ for $\epsilon=0,0.5$, and 1

The Hall effect seems to inhibit the creation of net magnetic helicity by the dynamo process, as can be seen from Figure 14. We saw earlier that the MHD dynamo is an efficient generator of magnetic helicity with $\langle\boldsymbol{A} \cdot \boldsymbol{B}\rangle$ / $\left\langle B^{2}\right\rangle \approx-0.4$. Most of this magnetic helicity is concentrated at large scales. However, in the presence of the Hall effect, the net magnetic helicity oscillates with $\langle\boldsymbol{A} \cdot \boldsymbol{B}\rangle /$ $\left\langle B^{2}\right\rangle<-0.05$ for the runs with both $\epsilon=0.5$ and 1 . Even when the Hall effect is marginal and acts only close to the dissipation scales $(\epsilon=0.066)$, the generation of mean magnetic helicity decreases by a factor of 2 . This strong difference in the evolution of the magnetic helicity between the MHD and the Hall-MHD dynamos is in good agreement with previous theoretical results (Ji 1999), which predict that MHD turbulent dynamo action converts magnetic helicity from the turbulent field to the mean field, but in the presence of the Hall effect, the magnetic helicity is only transported across space.

In Hall-MHD, equation (18) is still valid. Therefore, the dynamo process must create equal and opposite amounts of magnetic helicity at large (with the same sign as the $\alpha$ coefficient) and small scales (but with opposite sign). However, in the presence of the Hall effect, the reconnection events in the small scale are likely to be faster (Priest \& Forbes 1998) and therefore dissipate less magnetic helicity (Freedman \& Berger 1993). The Hall effect even allows, in principle, reconnection without dissipation, and for such

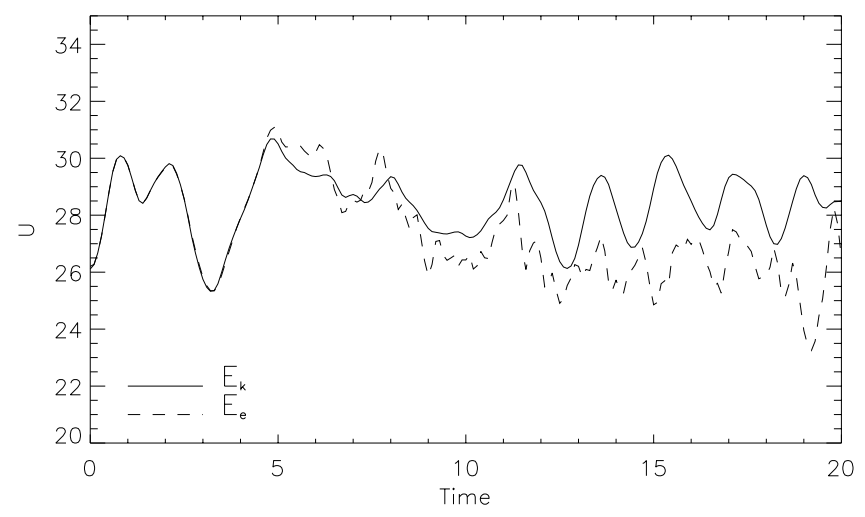

FIG. 12.-Kinetic energy $E_{k}$ and electronic kinetic energy $E_{e}=\left\langle u_{e}^{2}\right\rangle$ for $\epsilon=1$.

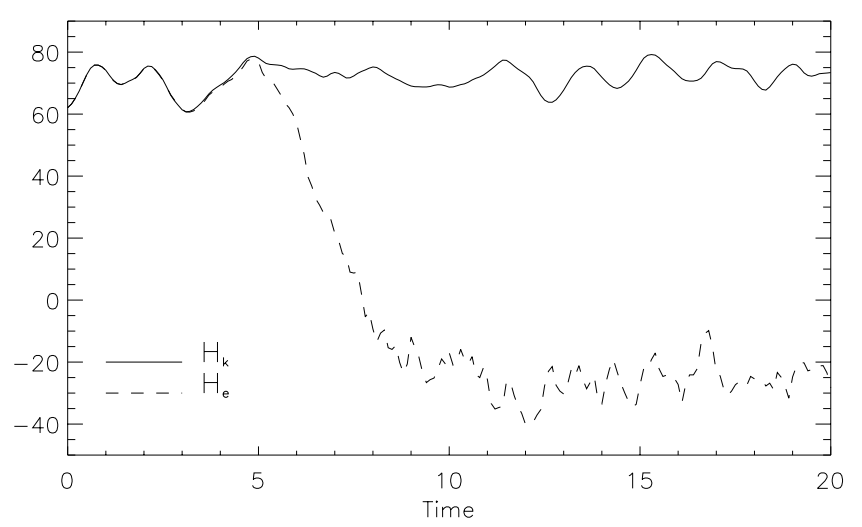

FIG. 13.-Kinetic helicity $H_{k}$ and electronic kinetic helicity $H_{e}$ for $\epsilon=1$.

processes the total magnetic helicity must be conserved (Wang, Bhattacharjee, \& Ma 2000). This relative excess of magnetic helicity at small scales (compared to the MHD case) cascades to larger length scales (Pouquet et al. 1976), canceling the initial large-scale magnetic helicity of opposite sign. This process can in principle explain the inefficiency of the Hall-MHD dynamo in generating magnetic helicity when compared to the MHD dynamo.

Figure 15 shows the spectrum of magnetic helicity for the MHD run and a Hall-MHD run with $\epsilon=1$. In good agreement with equation (18), the magnetic helicity is positive at small scales and negative at large scales. However, a large fraction of the small-scale magnetic helicity is destroyed by dissipation before it can cascade to larger scales. On the other hand, in the Hall-MHD run the amount of positive and negative helicity at small and large scales remains almost equal.

Note that for this cancellation of net helicity, the Hall effect is not required to act at all scales (as for the runs with $\epsilon<0.5$ ). Even an almost classical MHD dynamo (see, e.g., the results when $\epsilon=0.066$ ), with reconnection events taking place at scales such that kinetic effects cannot be neglected, could substantially decrease the generation of magnetic helicity by the $\alpha$-effect. This result might be important in dynamos where the generation of magnetic helicity is currently being discussed, such as the solar dynamo and reconnection events taking place in the solar corona.

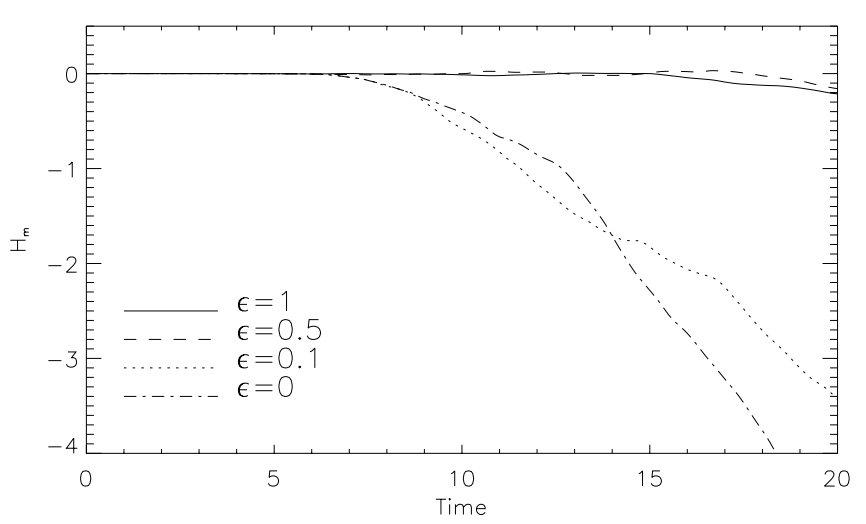

FIG. 14.-Magnetic helicity for $\epsilon=0,0.1,0.5$, and 1 

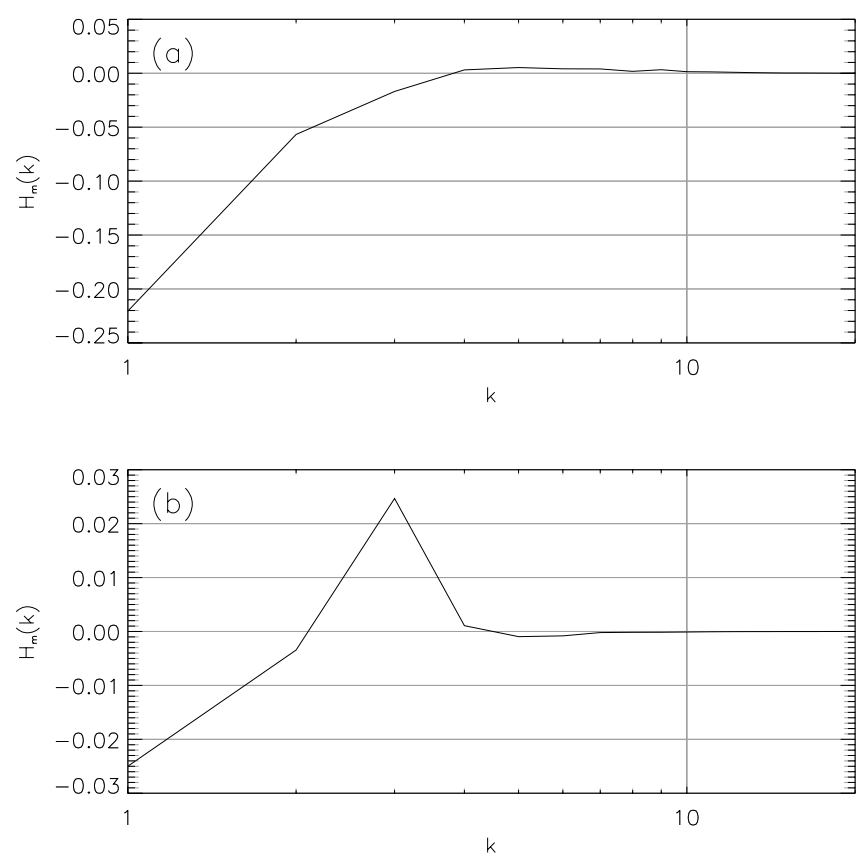

FIG. 15.-Magnetic helicity spectrum for $(a)$ the MHD run and $(b)$ a Hall-MHD run.

\section{DISCUSSION}

We present results of the first numerical simulations of Hall-MHD turbulent dynamo action. The main theme of this preliminary effort is to compare and contrast the HallMHD dynamo with the conventional MHD dynamo operating under the same physical conditions (Reynolds numbers, initial kinetic energy, and magnetic seed). The code is tested against available literature to make sure that when the Hall effect is switched off, previous results (for example Meneguzzi et al. 1981; Brandenburg 2001) are readily reproduced. By calculating the magnitude and nature of the generated magnetic field as the amplitude of the Hall term is varied, we show that the Hall-MHD dynamo can be fundamentally different from its classical MHD counterpart.

The simulations demonstrate that depending on the location of the Hall length scale with respect to the forcing ( $\left.L_{\text {force }}\right)$, seed $\left(L_{\text {seed }}\right)$, and dissipation lengths, the generation of the magnetic energy can strongly increase or decrease. While the MHD dynamo reaches equipartition, the final state of the Hall-MHD dynamo depends on $\epsilon$, the measure of the strength of the Hall term. Three different regimes can be distinguished: (1) the new results are only marginally different from MHD, (2) the generation of magnetic energy is substantially enhanced (Hall-enhanced dynamo), or (3) it is substantially inhibited (Hall-suppressed dynamo). A nontrivial result is that the efficiency of the dynamo does not scale monotonically with $\epsilon$.

The results pertaining to the changes of dynamo efficiency with $\epsilon$ are summarized in Figure 16, in which the maximum attained value of the magnetic energy is plotted as a function of $\epsilon$. The three different regimes of the Hall-MHD dynamo can be clearly identified. For reference, the maximum value of the magnetic energy obtained in the MHD run is also shown. Note that in the Hall-enhanced case, the maximum magnetic energy can be twice the energy gener-

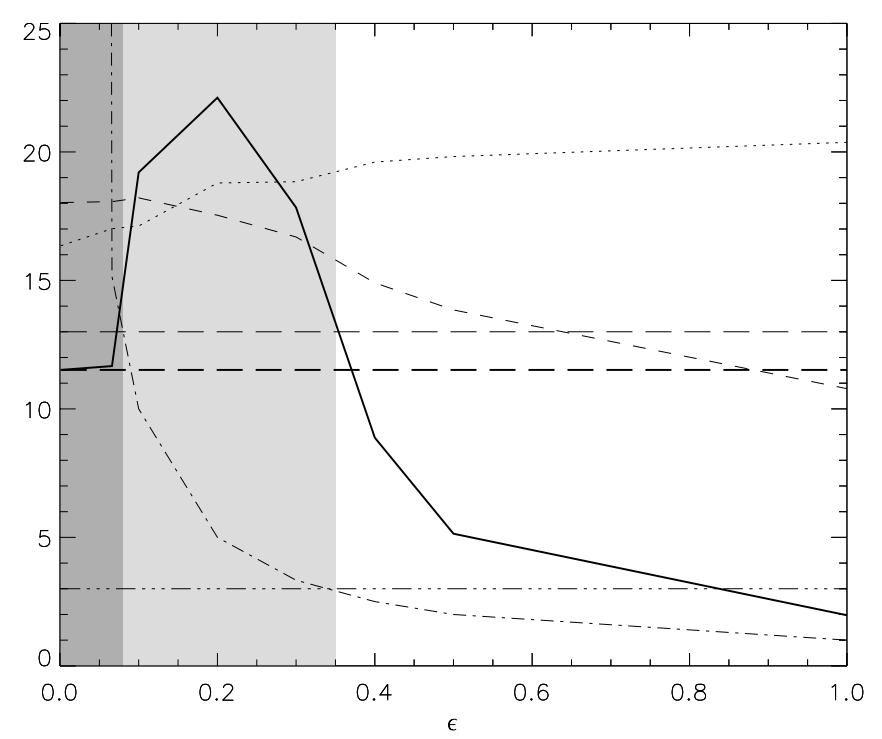

FIG. 16.-Efficiency of Hall-MHD dynamos. The thick lines correspond to magnetic energy, while the thin lines correspond to wavenumbers $k$. The thick dashed line is shown as a reference for the maximum value of magnetic energy in the MHD simulation $(\epsilon=0)$, while the thick solid line shows the maximum value reached by the magnetic energy as a function of $\epsilon$ for several Hall-MHD runs. The dotted line corresponds to the kinetic dissipation wavenumber $k_{\nu}$, and the dashed line shows the magnetic dissipation wavenumber $k_{\eta}$. The long-dashed line shows the wavenumber at which the initial magnetic field is located $\left(k_{\text {seed }}=13\right.$ in all the runs), and the tripledot-dashed line shows the forcing wavenumber $\left(k_{\text {force }}=3\right.$ in all the runs). The dot-dashed line corresponds to the Hall wavenumber $\left(k_{\mathrm{Hall}}=1 / \epsilon\right)$. The shaded area corresponds to the Hall-dominated microscale dynamo, where $k_{\text {seed }}>k_{\text {Hall }}>k_{\text {force }}$. The dark shaded area satisfies $\epsilon \rightarrow 0$ and $k_{\text {Hall }}>k_{\text {seed }}$, and the magnetic energy tends to the MHD value. Finally, in the white area, $\epsilon \rightarrow 1$ and $k_{\text {Hall }}<k_{\text {force }}$ (massive Hall dynamo).

ated in the MHD case, while in the Hall-suppressed regime, the energy could drop by 1 order of magnitude. The characteristic inverse length scales (magnetic and kinetic dissipation wavenumbers, forcing wavenumber, and seed wavenumber) are also shown for clarity. Note that the generation of the magnetic field is more efficient than in the MHD case in the region where $L_{\text {seed }}<L_{\text {Hall }}<L_{\text {force. }}$. When $L_{\text {Hall }}<L_{\text {seed }}$ and $L_{\text {Hall }}$ approaches the dissipation scale, the magnetic energy asymptotically tends to the MHD value. Finally, when $L_{\text {Hall }}>L_{\text {force }}$ the dynamo action becomes less efficient.

The observed dependence of the saturation level of the dynamo on the strength of the Hall term $(\epsilon)$ has important implications for astrophysics. Since $\epsilon$ is determined by the physical parameters of the astrophysical environment in which the dynamo operates (such as the masses of the charge carriers and their densities), the MHD estimates for the maximum magnetic energy generated by some objects could be way off the mark, and the inclusion of the Hall effect becomes crucial. These conclusions are qualitatively in good agreement with recent theoretical predictions (Mininni et al. 2002).

We want to emphasize that current numerical simulations such as the ones reported here are far away from realistic Reynolds numbers for astrophysical plasmas. More work must be carried out to study how the separation between the relevant scales of the system affects these results. Nonetheless, we discuss potential applications of our results to real astrophysical systems, keeping in mind this important shortcoming. As shown in $\S 1$, typical values for a 
protostellar disk (Balbus \& Terquem 2001) yield $H / O \approx 10^{2}$ and $H / I \approx 10^{-4}$. These values imply that the Hall scale in this case is larger than the dissipation scale by 2 orders of magnitude but smaller than the largest scales of the system. Therefore, we expect an enhancement of the dynamo mechanism by the Hall currents. On the other hand, in some dwarf nova disks and protoplanetary disks, $H / I \approx 1$ or even larger (Sano \& Stone 2002). Therefore, an inhibition of the dynamo is expected for these objects.

The final result of this preliminary study is that although the generation of a large-scale magnetic field was observed in all cases, the topological properties of the generated field could vary. Regardless of suppression or enhancement of the magnetic energy, the Hall effect in our simulations always and strongly suppresses the production of net magnetic helicity. This result, extremely relevant to helicitygeneration schemes, is in good agreement with previous theoretical results (Ji 1999).

The research of S. M. M. was supported by US Department of Energy contract DE-FG03-96ER-54366. The research of D. O. G. and P. D. M. has been funded by grant X209/01 from the University of Buenos Aires. P. D. M. is a fellow of CONICET, and D. O. G. is a member of the Carrera del Investigador Cientifico of CONICET.
Balbus, S. A., \& Terquem, C. 2001, ApJ, 552, 235

Brandenburg, A. 2001, ApJ, 550, 824

Canuto, C., Hussaini, M. Y., Quarteroni, A., \& Zang, T. A. 1988, Spectral Methods in Fluid Dynamics (Berlin: Springer)

Cattaneo, F., \& Hughes, D. W. 1996, Phys. Rev. E, 54, R4532

Freedman, M. H., \& Berger, M. A. 1993, Geophys. Astrophys. Fluid Dyn., 73,91

Galanti, B., Kleeorin, N., \& Rogachevskii, I. 1994, Phys. Plasmas, 1, 3843

Gruzinov, A., \& Diamond, P. H. 1994, Phys. Rev. Lett., 72, 1651

Heintzmann, H. 1983, J. Exp. Theor. Phys., 57, 251

Hollerbach, R., \& Rüdiger, G. 2002, MNRAS, 337, 216

Ji, H. 1999, Phys. Rev. Lett., 83, 3198

Kazantsev, A. P. 1968, J. Exp. Theor. Phys., 26, 1031

Meneguzzi, M., Frisch, U., \& Pouquet, A. 1981, Phys. Rev. Lett., 47, 1060

Mininni, P. D., Gómez, D. O., \& Mahajan, S. M. 2002, ApJ, 567, L81 2003, ApJ, 584, 1120

\section{REFERENCES}

Norman, C., \& Heyvaerts, J. 1985, A\&A, 147, 247

Orzag, S. A., \& Patterson, G. S. 1972, Phys. Rev. Lett., 28, 76

Potekhin, A. Y. 1999, A\&A, 351, 787

Pouquet, A., Frisch, U., \& Leorat, J. 1976, J. Fluid Mech., 77, 321

Priest, E., \& Forbes, T. 1998, Magnetic Reconnection (Cambridge: Cambridge Univ. Press)

Sano, T., \& Stone, J. M. 2002, ApJ, 570, 314

Seehafer, N. 1996, Phys. Rev. E, 53, 1283

Shalybkov, D. A., \& Urpin, V. A. 1997, A\&A, 321, 685

Shu, F., Najita, J., Ostriker, E., \& Wilkin, F. 1994, ApJ, 429, 781

Tajima, T., Cable, S., Shibata, K., \& Kulsrud, R. M. 1992, ApJ, 390, 309

Urpin, V. A., \& Yakovlev, D. 1980, AZh, 24, 303

Wang, X., Bhattacharjee, A., \& Ma, Z. W. 2000, J. Geophys. Res., 105, 27633

Wardle, M. 1999, MNRAS, 307, 849

Wardle, M., \& Ng, C. 1999, MNRAS, 303, 239 